\title{
Four-membered Group 13 Metal(I) N-Heterocyclic Carbene Analogues: Synthesis, Characterization and Theoretical Studies
}

\author{
Cameron Jones,* Peter C. Junk, Jamie A. Platts and Andreas Stasch
}

\section{SUPPORTING INFORMATION}

Synthetic details for $\mathbf{1}-\mathbf{3}$ (spectroscopic data can be found in the main text)

NB: The guanidine, $(\mathrm{Ar}) \mathrm{N}(\mathrm{H}) \mathrm{C}\left(\mathrm{NCy}_{2}\right)=\mathrm{N}(\mathrm{Ar})$, GisoH, $\mathrm{Ar}=\mathrm{C}_{6} \mathrm{H}_{3} \operatorname{Pr}_{2}^{\mathrm{i}}-2,6$; Cy $=$ cyclohexyl, was prepared by treating the carbodimide, $\mathrm{ArN}=\mathrm{C}=\mathrm{NAr},{ }^{1}$ with one equivalent of $\mathrm{Li}\left[\mathrm{NCy}_{2}\right]$ in $\mathrm{THF}$, followed by aqueous work-up. Recrystallization of the crude product from hexane yields GisoH in yields greater than $90 \%$. Full synthetic and spectroscopic data for this compound will be reported in a future publication.

Compound 1, [Ga(Giso)]: $\mathrm{Bu}^{\mathrm{n}} \mathrm{Li}(0.95 \mathrm{~mL}$ of a $1.6 \mathrm{M}$ solution in hexanes, $1.52 \mathrm{mmol})$ was added to a solution of GisoH $(0.800 \mathrm{~g}, 1.47 \mathrm{mmol})$ in toluene $(20 \mathrm{~mL})$ over 5 mins. The resultant solution was stirred for $1 \mathrm{~h}$ and then reacted with a slurry of "GaI" ${ }^{2}$ at $-78^{\circ} \mathrm{C}$ (prepared by reacting Ga $(126 \mathrm{mg}, 1.81 \mathrm{mmol})$ with $\mathrm{I}_{2}(219 \mathrm{mg}, 0.863 \mathrm{mmol})$ under ultrasonic conditions in toluene $(20 \mathrm{~mL})$ for 2 hours at $30^{\circ} \mathrm{C}$ ). The reaction mixture was warmed to room temperature over 1 hour. After a further 18 hours it was filtered and volatiles removed from the filtrate under reduced pressure. The residue was extracted into hexane $(30 \mathrm{~mL})$ and filtered. The filtrate was concentrated to $c a .15 \mathrm{~mL}$ and slowly cooled to $-30{ }^{\circ} \mathrm{C}$ to yield colourless crystals of $\mathbf{1}(0.32 \mathrm{~g}, 35 \%)$.

Compound 2, [In(Giso)]: $\mathrm{Bu}^{\mathrm{n}} \mathrm{Li}(0.90 \mathrm{~mL}$ of a $1.6 \mathrm{M}$ solution in hexanes, $1.44 \mathrm{mmol})$ was added to a solution of GisoH $(0.745 \mathrm{~g}, 1.37 \mathrm{mmol})$ in toluene $(20 \mathrm{~mL})$ over 5 mins. The resultant solution was stirred for $1 \mathrm{~h}$, cooled to $-78^{\circ} \mathrm{C}$ and treated with $\mathrm{InCl}(0.255 \mathrm{~g}, 1.70 \mathrm{mmol})$. The reaction mixture was warmed to $25^{\circ} \mathrm{C}$ and stirred for 18 hours, after which it was filtered and volatiles removed from the filtrate under reduced pressure. The residue was extracted into hexane $(30 \mathrm{~mL})$, the extract concentrated to $\mathrm{ca}$. $12 \mathrm{~mL}$ and slowly cooled to $-30{ }^{\circ} \mathrm{C}$ to yield colourless crystals of 2 $(0.42 \mathrm{~g}, 48 \%)$.

Compound 3, [TI(Giso)]: $\mathrm{Bu}^{\mathrm{n}} \mathrm{Li}(0.90 \mathrm{~mL}$ of a $1.6 \mathrm{M}$ solution in hexanes, $1.44 \mathrm{mmol})$ was added to a solution of $\mathrm{GisoH}(0.760 \mathrm{~g}, 1.40 \mathrm{mmol})$ in toluene $(20 \mathrm{~mL})$ over 5 mins. The resultant solution was stirred for $1 \mathrm{~h}$, cooled to $-78^{\circ} \mathrm{C}$ and treated with $\mathrm{TlBr}(0.49 \mathrm{~g}, 1.72 \mathrm{mmol})$. The reaction mixture was warmed to $25^{\circ} \mathrm{C}$ and stirred for 18 hours, after which it was filtered and volatiles removed from the filtrate under reduced pressure. The residue was extracted into hexane $(30 \mathrm{~mL})$, the extract concentrated to $c a .5 \mathrm{~mL}$ and slowly cooled to $-30{ }^{\circ} \mathrm{C}$ to yield yellow crystals of 3 $(0.71 \mathrm{~g}, 68 \%)$.

N.B.: Although the syntheses of compounds $\mathbf{1}-\mathbf{3}$ are carried out with in-situ generated Li[Giso], this reagent can be isolated prior to its reaction with metal(I) halides as a crystalline solid. There appears to be no yield advantage in using in-situ generated or isolated Li[Giso] in the synthesis of 1 - 3. Selected spectroscopic data for Li[Giso] can be found below.

${ }^{1} \mathrm{H}$ NMR $\left(300 \mathrm{MHz}, 296 \mathrm{~K}, \mathrm{C}_{6} \mathrm{D}_{6}\right): \delta 7.26-6.94(\mathrm{~m}, 6 \mathrm{H}, \mathrm{ArH}), 3.57$ (sept, $J=6.8 \mathrm{~Hz}, 4 \mathrm{H}$, $\left.\mathrm{CH}\left(\mathrm{CH}_{3}\right)_{2}\right), 3.35$ (broad, $\left.2 \mathrm{H}, \mathrm{CHN}\right), 2.03$ (broad, $\left.4 \mathrm{H}, \mathrm{CH}{ }_{2}\right) 1.78-1.48\left(\mathrm{~m}, 8 \mathrm{H}, \mathrm{CH}_{2}\right), 1.49(\mathrm{~d}, J=$ $\left.6.8 \mathrm{~Hz}, 12 \mathrm{H}, \mathrm{CH}\left(\mathrm{CH}_{3}\right)_{2}\right), 1.18\left(\mathrm{~d}, J=6.8 \mathrm{~Hz}, 12 \mathrm{H}, \mathrm{CH}\left(\mathrm{CH}_{3}\right)_{2}\right), 1.17-0.85\left(\mathrm{~m}, 8 \mathrm{H}, \mathrm{CH}_{2}\right) ;{ }^{7} \mathrm{Li} \mathrm{NMR}$ $\left(155.5 \mathrm{MHz}, 296 \mathrm{~K}, \mathrm{C}_{6} \mathrm{D}_{6}\right): \delta 2.6(\mathrm{~s}) ;{ }^{13} \mathrm{C} \mathrm{NMR}\left(75.5 \mathrm{MHz}, 296 \mathrm{~K}, \mathrm{C}_{6} \mathrm{D}_{6}\right): \delta 160.6$ (broad, $\left.C_{\mathrm{N}}\right)$, 150.2 (broad, $\mathrm{ArC}$ ), $141.3(\mathrm{ArC}), 124.0(\mathrm{ArC}), 120.8(\mathrm{ArC}), 58.6(\mathrm{HCN}), 35.1\left(\mathrm{CH}_{2}\right), 28.7$ 
$\left(\mathrm{CH}\left(\mathrm{CH}_{3}\right), 27.9\left(\mathrm{CH}\left(\mathrm{CH}_{3}\right)\right), 26.9\left(\mathrm{CH}\left(\mathrm{CH}_{3}\right)\right), 25.0\left(\mathrm{CH}_{2}\right), 23.7\left(\mathrm{CH}_{2}\right)\right.$; IR (Nujol): v: 1612 (s), 1583 (s), 1236 (s), 1156 (m), 1110 (m), 1027 (m), 933 (m), 895 (m), 792 (m), 748 (m).

1. Ogawa, K.; Akazawa, M. Japanese Patent Appl. JP 91-208987910517.

2. Green, M.L.H.; Mountford, P.; Smout, G.J.; Speel, S.R. Polyhedron 1990, 9, 2763. 


\section{Discussion of the molecular structure of $\left[\mathrm{TI}\left(\eta^{1}-\mathrm{N}: \eta^{3}-\mathrm{Ar}-\mathrm{Giso}\right)\right] 3$}

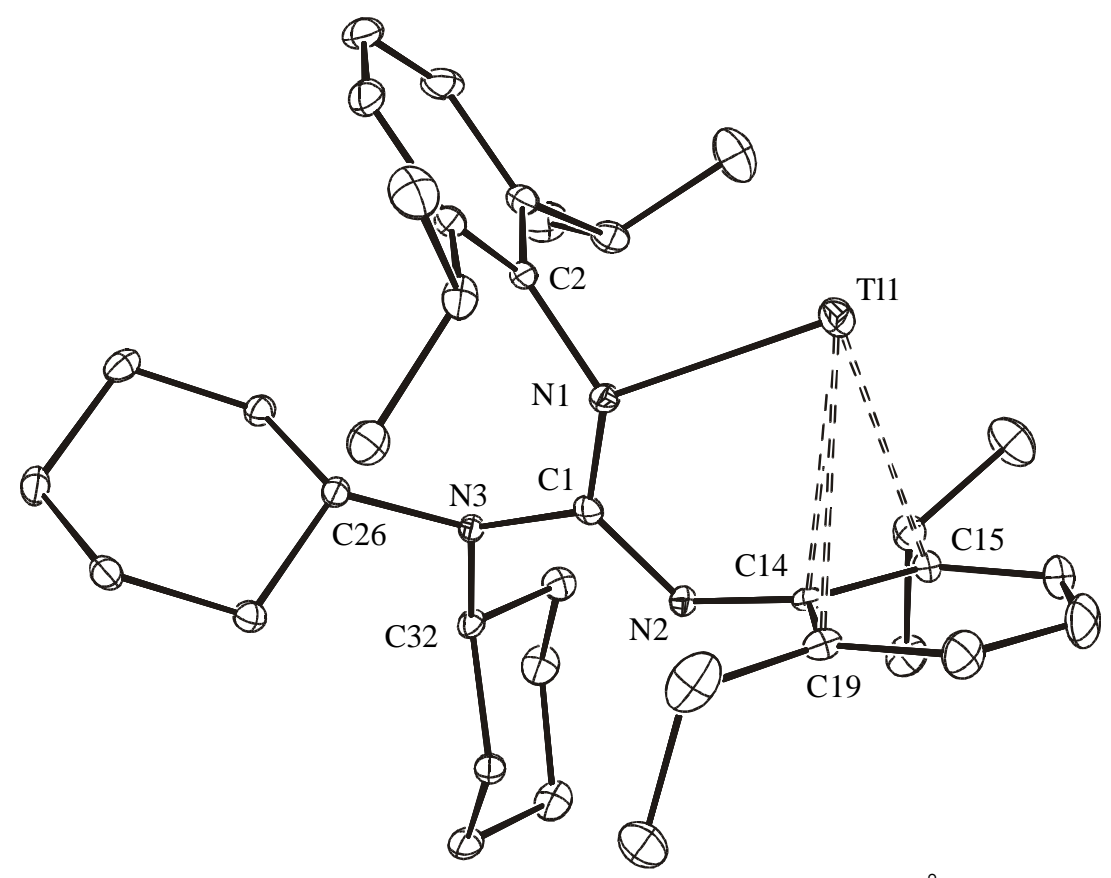

Figure 1-S: Molecular structure of 3. Relevant bond lengths $(\AA)$ and angles $\left({ }^{\circ}\right)$ : $\mathrm{Tl}(1)-\mathrm{N}(1)$ 2.460(3), N(1)-C(1) 1.370(4), N(2)-C(1) 1.309(4), N(3)-C(1) 1.408(4), Tl(1)-C(14) 2.927(3), Tl(1)$\mathrm{C}(15) \quad 3.181(3), \quad \mathrm{Tl}(1)-\mathrm{C}(19) \quad 3.052(3), \quad \mathrm{C}(1)-\mathrm{N}(1)-\mathrm{Tl}(1) \quad 124.1(2), \quad \mathrm{C}(2)-\mathrm{N}(1)-\mathrm{Tl}(1)$ 104.49(17), C(1)-N(1)-C(2) 124.7(3), N(2)-C(1)-N(1) 122.2(3), N(2)-C(1)-N(3) 116.1(3), N(1)$\mathrm{C}(1)-\mathrm{N}(3) 121.7(3)$.

The molecular structure of compound $\mathbf{3}$ shows it to be monomeric with its thallium center coordinated in an $\eta^{1}$-fashion by the amido center $(\mathrm{N}(1))$ of the Giso ligand. The metal also has an $\eta^{3}$-interaction with the aryl substituent of $\mathrm{N}(2)$. The magnitudes of the $\mathrm{C}(1)-\mathrm{N}$ bond lengths of the guanidinate ligand suggest a low degree of delocalization over its $\mathrm{N}_{3} \mathrm{C}$ backbone. The structure of 3 is closely related to those of the amidinate complexes, $\left[\mathrm{M}\left(\eta^{1}-\mathrm{N}, \eta^{3}-\mathrm{Ar}-\mathrm{Piso}\right)\right], \mathrm{M}=\mathrm{In}$ or Tl, Piso = $\left[(\mathrm{Ar}) \mathrm{NC}\left(\mathrm{Bu}^{t}\right) \mathrm{N}(\mathrm{Ar})\right]^{-}$(see main text for reference), except that the metal centers of the latter are also weakly coordinated by one aryl group of an amidine molecule (PisoH) of crystallization. In contrast, the structure of $\mathbf{3}$ displays weak intermolecular interactions $(3.68-3.82 \AA)$ between the Tl-coordinated aryl substituent of one molecule and the $\mathrm{Tl}$ center of its nearest neighbor. 


\section{Supplementary Calculation Data for $\left[\mathbf{M}\left\{\eta^{2}-\mathbf{N}, \mathbf{N}^{\prime}-(\mathbf{P h}) \mathbf{N C}\left(\mathbf{N M e}_{2}\right) \mathbf{N}(\mathbf{P h})\right\}\right], \mathbf{M}=\mathbf{A l}$, Ga or In}

Geometries of molecules were optimised using the Gaussian03 package [a] using the methods recommended by Boehme and Frenking [b], namely the BP86 density functional [c] with a 631G(d) basis set on C, N and H [d] and Stuttgart-Dresden ECP/basis set augmented by a d-type polarisation function with exponent 0.207 on $\mathrm{Al}, \mathrm{Ga}$ and $\mathrm{In}$ [e, f]. All geometries were characterised as true minima via harmonic frequency calculation. Atomic charges, orbital populations, and bonding analysis were obtained from the NBO scheme [g].

[a] Frisch, M. J.; Trucks, G. W.; Schlegel, H. B.; Scuseria, G. E.; Robb, M. A.; Cheeseman, J. R.; Montgomery, Jr., J. A.; Vreven, T.; Kudin, K. N.; Burant, J. C.; Millam, J. M.; Iyengar, S. S.; Tomasi, J.; Barone, V.; Mennucci, B.; Cossi, M.; Scalmani, G.; Rega, N.; Petersson, G. A.; Nakatsuji, H.; Hada, M.; Ehara, M.; Toyota, K.; Fukuda, R.; Hasegawa, J.; Ishida, M.; Nakajima, T.; Honda, Y.; Kitao, O.; Nakai, H.; Klene, M.; Li, X.; Knox, J. E.; Hratchian, H. P.; Cross, J. B.; Adamo, C.; Jaramillo, J.; Gomperts, R.; Stratmann, R. E.; Yazyev, O.; Austin, A. J.; Cammi, R.; Pomelli, C.; Ochterski, J. W.; Ayala, P. Y.; Morokuma, K.; Voth, G. A.; Salvador, P.; Dannenberg, J. J.; Zakrzewski, V. G.; Dapprich, S.; Daniels, A. D.; Strain, M. C.; Farkas, O.; Malick, D. K.; Rabuck, A. D.; Raghavachari, K.; Foresman, J. B.; Ortiz, J. V.; Cui, Q.; Baboul, A. G.;. Clifford, S; Cioslowski, J.; Stefanov, B. B.; Liu, G.; Liashenko, A.; Piskorz, P.; Komaromi, I.; Martin, R. L.; Fox, D. J.; Keith, T.; Al-Laham, M. A.; Peng, C. Y.; Nanayakkara, A.; Challacombe, M.; Gill, P. M. W.; Johnson, B.; Chen, W.; Wong, M. W.; Gonzalez, C.; Pople, J. A. Gaussian 03, Revision B.05, Gaussian, Inc., Pittsburgh PA, 2003.

[b] Boehme, C.; Frenking, G. Chem. Eur. J. 1999, 5, 7.

[c] i) Becke, A.D. Phys. Rev. A 1988, 38, 3098. ii) Perdew, J.P. Phys. Rev. A 1986, 33, 8822.

[d] i) Ditchfield, R.; Hehre, W.J.; Pople, J.A. J. Chem. Phys. 1971, 54, 724, ii) Hehre, W.J.; Ditchfield, R.; Pople, J.A. J. Chem. Phys. 1972, 56, 2257, iii) Gordon, M.S. Chem. Phys. Lett. 1980, 76, 163, iv) Hariharan, P.C.; Pople, J.A. Theor. Chim. Acta 1973, $28,213$.

[e] i) Dolg, M.; Wedig, U.; Stoll, H.; Preuss, H. J. Chem. Phys. 1987, 86, 866, ii) Bergner, A.; Dolg, M.; Kuehle, W.; Stoll, H.; Preuss, H. Mol. Phys. 1993, 80, 1431.

[f] Andzelm, J.; Huzinaga, S.; Klobukowski, M.; Radzio, E.; Sakai, Y.; Tatekawi, H. Gaussian Basis Sets for Molecular Calculations, Elsevier, Amsterdam, 1984.

[g] Reed, A. E.; Curtiss, L. A.; Weinhold, F. Chem. Rev. 1988, 88, 899. 
Summary of fully optimized geometries of $\left[: M\left\{\eta^{2}-N_{,} N^{\prime}-(P h) N C\left(N M e_{2}\right) N(P h)\right\}\right], M=A l-I n$

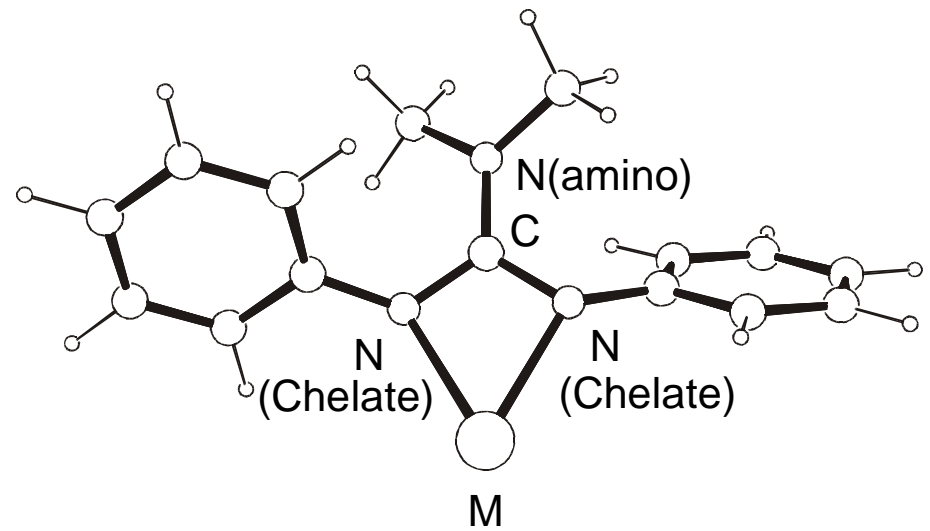

$\mathrm{M}$

$\mathrm{Al}$

$\mathrm{Ga}$

In

M-N(chelate) $\AA$ (mean)

2.078

2.207

2.332

C-N(chelate) $\AA$ (mean)

1.362

1.359

1.360

C-N(amino) $\AA$

1.374

1.380

1.383

N-M-N deg.

65.16

61.67

58.41

N(chelate)-C-N(chelate) deg.

110.50

112.66

113.62

$\mathrm{N}$ (chelate)-C-N(amino) deg. (mean)

124.74

123.67

123.19

$\Sigma$ angles at N(amino) deg.

360.00

360.00

360.00

$\Sigma$ angles at N(chelate)deg. (mean)

349.92

349.00

349.42 
Coordinate file (.xyz) for the fully optimized geometry of $\left[\mathrm{Al}\left\{\eta^{2}-\mathbf{N}, \mathbf{N}^{\prime}-(\mathrm{Ph}) \mathbf{N C}\left(\mathrm{NMe}_{2}\right) \mathbf{N}(\mathrm{Ph})\right\}\right]$ total energy -987.925257

$\begin{array}{rrrr}\mathrm{C} & 3.830401 & 1.331690 & -3.306566 \\ \mathrm{C} & 3.646100 & 0.920967 & -1.975447 \\ \mathrm{C} & 4.705959 & 1.055868 & -1.062860 \\ \mathrm{C} & 5.938318 & 1.580628 & -1.475727 \\ \mathrm{C} & 6.136237 & 1.987182 & -2.815908 \\ \mathrm{C} & 5.056935 & 1.866678 & -3.726162 \\ \mathrm{~N} & 7.375092 & 2.578342 & -3.152512 \\ \mathrm{C} & 7.960839 & 2.560198 & -4.381531 \\ \mathrm{~N} & 7.834929 & 1.540754 & -5.294201 \\ \mathrm{C} & 8.031325 & 1.753148 & -6.727408 \\ \mathrm{Al} & 8.192266 & 4.434978 & -2.703175 \\ \mathrm{~N} & 8.688779 & 3.693684 & -4.579541 \\ \mathrm{C} & 9.867457 & 3.797432 & -5.352505 \\ \mathrm{C} & 10.801467 & 2.741436 & -5.495597 \\ \mathrm{C} & 11.985765 & 2.934317 & -6.221471 \\ \mathrm{C} & 12.273167 & 4.179783 & -6.805210 \\ \mathrm{C} & 11.360513 & 5.236885 & -6.650573 \\ \mathrm{C} & 10.169916 & 5.050398 & -5.934864 \\ \mathrm{C} & 7.496367 & 0.176337 & -4.892310 \\ \mathrm{H} & 12.697517 & 2.105861 & -6.318103 \\ \mathrm{H} & 13.201927 & 4.326464 & -7.367034 \\ \mathrm{H} & 11.575012 & 6.216634 & -7.092811 \\ \mathrm{H} & 4.574433 & 0.747916 & -0.019137 \\ \mathrm{H} & 2.684498 & 0.508274 & -1.651776 \\ \mathrm{H} & 3.006211 & 1.249699 & -4.025076 \\ \mathrm{H} & 7.194168 & 1.279859 & -7.274521 \\ \mathrm{H} & 8.036241 & 2.829270 & -6.949445 \\ \mathrm{H} & 8.984013 & 1.316668 & -7.083413 \\ \mathrm{H} & 8.188594 & -0.524391 & -5.396263 \\ \mathrm{H} & 7.610801 & 0.066760 & -3.804992 \\ \mathrm{H} & 6.457027 & -0.089118 & -5.164659 \\ \mathrm{H} & 10.601535 & 1.777260 & -5.014674 \\ \mathrm{H} & 9.452473 & 5.869855 & -5.815678 \\ \mathrm{H} & 5.180966 & 2.216304 & -4.757313 \\ \mathrm{H} & 6.769622 & 1.683176 & -0.769429\end{array}$

Coordinate file (.xyz) for the fully optimized geometry of [Ga $\left.\left\{\eta^{2}-\mathbf{N}, \mathbf{N}^{\prime}-(\mathrm{Ph}) \mathbf{N C}\left(\mathrm{NMe}_{2}\right) \mathbf{N}(\mathrm{Ph})\right\}\right]$ total energy -747.593369

$\begin{array}{rrrr}\mathrm{C} & 3.842589 & 1.326639 & -3.333079 \\ \mathrm{C} & 3.643299 & 0.901737 & -2.008565 \\ \mathrm{C} & 4.694664 & 1.027409 & -1.084486 \\ \mathrm{C} & 5.929906 & 1.558311 & -1.479253 \\ \mathrm{C} & 6.145352 & 1.981192 & -2.813302 \\ \mathrm{C} & 5.072949 & 1.866398 & -3.734816 \\ \mathrm{~N} & 7.385219 & 2.574862 & -3.126392 \\ \mathrm{C} & 7.964779 & 2.588380 & -4.355640 \\ \mathrm{~N} & 7.838714 & 1.564315 & -5.272323 \\ \mathrm{C} & 7.996450 & 1.782508 & -6.708153 \\ \mathrm{Ga} & 8.207180 & 4.552788 & -2.596024 \\ \mathrm{~N} & 8.682388 & 3.719764 & -4.583640 \\ \mathrm{C} & 9.858314 & 3.800451 & -5.357238 \\ \mathrm{C} & 10.785207 & 2.735199 & -5.493319 \\ \mathrm{C} & 11.969900 & 2.909998 & -6.223011 \\ \mathrm{C} & 12.268505 & 4.145691 & -6.821764 \\ \mathrm{C} & 11.364098 & 5.211714 & -6.677826 \\ \mathrm{C} & 10.174241 & 5.043558 & -5.957066 \\ \mathrm{C} & 7.537459 & 0.192906 & -4.869159\end{array}$




$\begin{array}{rrrr}\mathrm{H} & 12.673705 & 2.073677 & -6.311767 \\ \mathrm{H} & 13.197411 & 4.277653 & -7.387022 \\ \mathrm{H} & 11.585196 & 6.184358 & -7.132770 \\ \mathrm{H} & 4.553507 & 0.707152 & -0.045541 \\ \mathrm{H} & 2.678741 & 0.484802 & -1.699389 \\ \mathrm{H} & 3.026360 & 1.251082 & -4.061578 \\ \mathrm{H} & 7.155271 & 1.296038 & -7.238317 \\ \mathrm{H} & 7.977719 & 2.859293 & -6.927110 \\ \mathrm{H} & 8.948193 & 1.364498 & -7.089436 \\ \mathrm{H} & 8.232791 & -0.493553 & -5.389317 \\ \mathrm{H} & 7.676418 & 0.082881 & -3.784558 \\ \mathrm{H} & 6.497981 & -0.094659 & -5.119630 \\ \mathrm{H} & 10.577079 & 1.776871 & -5.004285 \\ \mathrm{H} & 9.464014 & 5.870979 & -5.847252 \\ \mathrm{H} & 5.207436 & 2.223508 & -4.762077 \\ \mathrm{H} & 6.753835 & 1.652797 & -0.762787\end{array}$

Coordinate file (.xyz) for the fully optimized geometry of $\left[\operatorname{In}\left\{\eta^{2}-\mathbf{N}, \mathbf{N}^{\prime}-(\mathbf{P h}) \mathbf{N C}\left(\mathbf{N M e}_{2}\right) \mathbf{N}(\mathbf{P h})\right\}\right]$ total energy -747.408271

$\begin{array}{rrrr}\mathrm{C} & 3.851740 & 1.313235 & -3.359724 \\ \mathrm{C} & 3.645864 & 0.864436 & -2.044108 \\ \mathrm{C} & 4.688944 & 0.985223 & -1.110057 \\ \mathrm{C} & 5.921624 & 1.535213 & -1.486506 \\ \mathrm{C} & 6.144614 & 1.982931 & -2.811566 \\ \mathrm{C} & 5.079768 & 1.871512 & -3.742890 \\ \mathrm{~N} & 7.379230 & 2.598662 & -3.103250 \\ \mathrm{C} & 7.967355 & 2.620476 & -4.328931 \\ \mathrm{~N} & 7.842208 & 1.594200 & -5.246893 \\ \mathrm{C} & 7.977480 & 1.813950 & -6.684316 \\ \mathrm{In} & 8.223836 & 4.681127 & -2.480746 \\ \mathrm{~N} & 8.691049 & 3.746921 & -4.566002 \\ \mathrm{C} & 9.857832 & 3.804181 & -5.356032 \\ \mathrm{C} & 10.777691 & 2.731077 & -5.482395 \\ \mathrm{C} & 11.956767 & 2.885298 & -6.225722 \\ \mathrm{C} & 12.257829 & 4.107795 & -6.849835 \\ \mathrm{C} & 11.360956 & 5.181611 & -6.717193 \\ \mathrm{C} & 10.176987 & 5.033758 & -5.982280 \\ \mathrm{C} & 7.564592 & 0.218194 & -4.844054 \\ \mathrm{H} & 12.654391 & 2.042869 & -6.305700 \\ \mathrm{H} & 13.182355 & 4.223656 & -7.425707 \\ \mathrm{H} & 11.583172 & 6.144382 & -7.192256 \\ \mathrm{H} & 4.543459 & 0.645969 & -0.077713 \\ \mathrm{H} & 2.683109 & 0.433029 & -1.749509 \\ \mathrm{H} & 3.042206 & 1.241451 & -4.096110 \\ \mathrm{H} & 7.130329 & 1.324437 & -7.202736 \\ \mathrm{H} & 7.951635 & 2.890714 & -6.902712 \\ \mathrm{H} & 8.924952 & 1.399795 & -7.080634 \\ \mathrm{H} & 8.267724 & -0.457230 & -5.368860 \\ \mathrm{H} & 7.710559 & 0.108455 & -3.760331 \\ \mathrm{H} & 6.528406 & -0.085795 & -5.089457 \\ \mathrm{H} & 10.567524 & 1.782216 & -4.976051 \\ \mathrm{H} & 9.472656 & 5.867751 & -5.882237 \\ \mathrm{H} & 5.218996 & 2.244800 & -4.763757 \\ \mathrm{H} & 6.739028 & 1.624899 & -0.761623\end{array}$




\section{Natural Population Analysis and Natural Electron Configuration for $\left[\mathrm{Al}\left\{\eta^{2}-\mathrm{N}, \mathrm{N}^{\prime}-\right.\right.$ (Ph)NC(NMe $\left.\left.\left.{ }_{2}\right) \mathbf{N}(\mathbf{P h})\right\}\right]$}

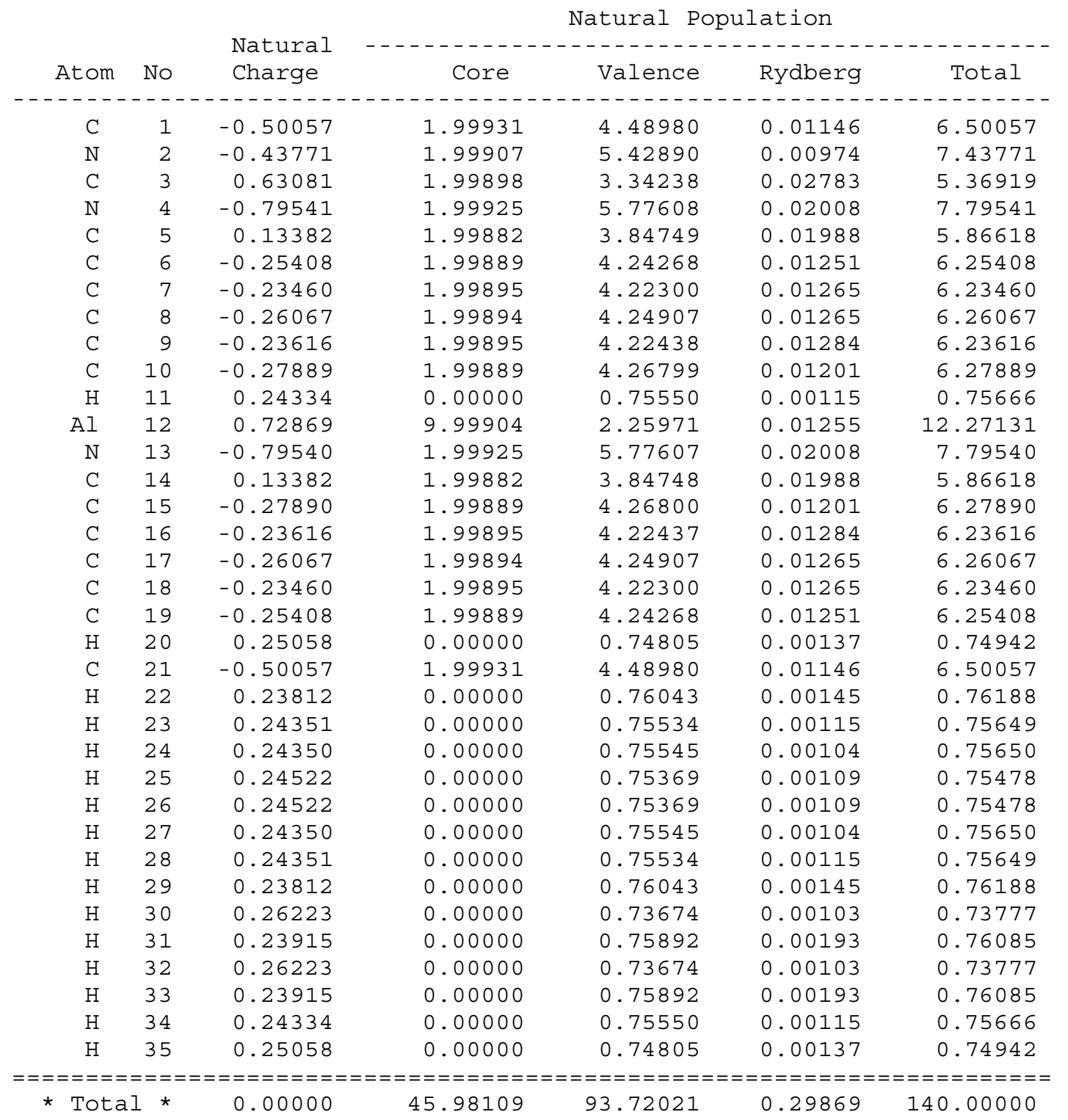

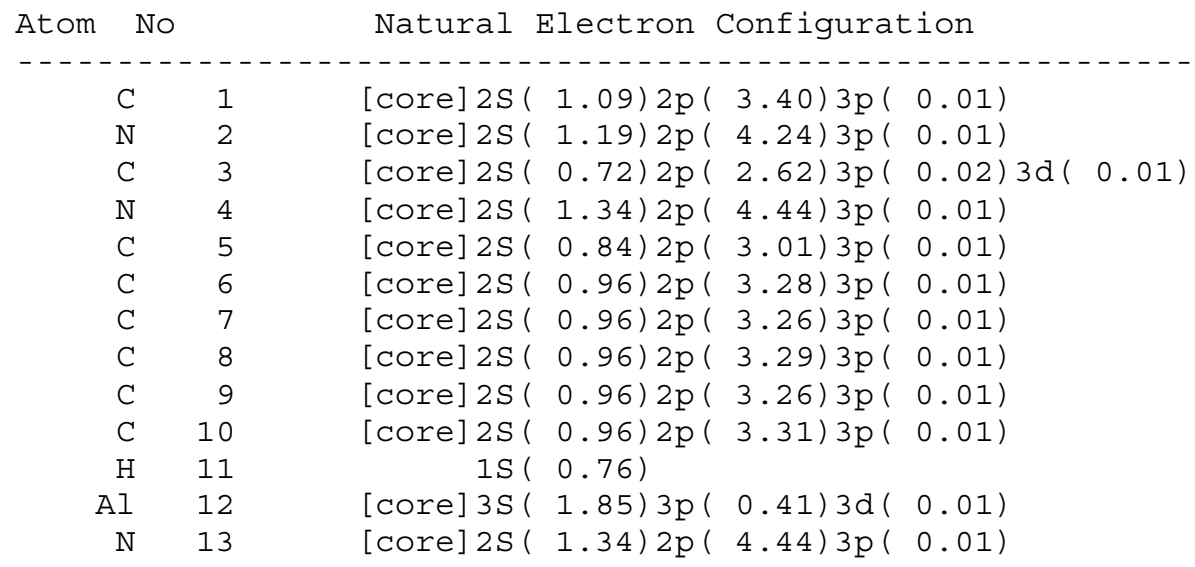




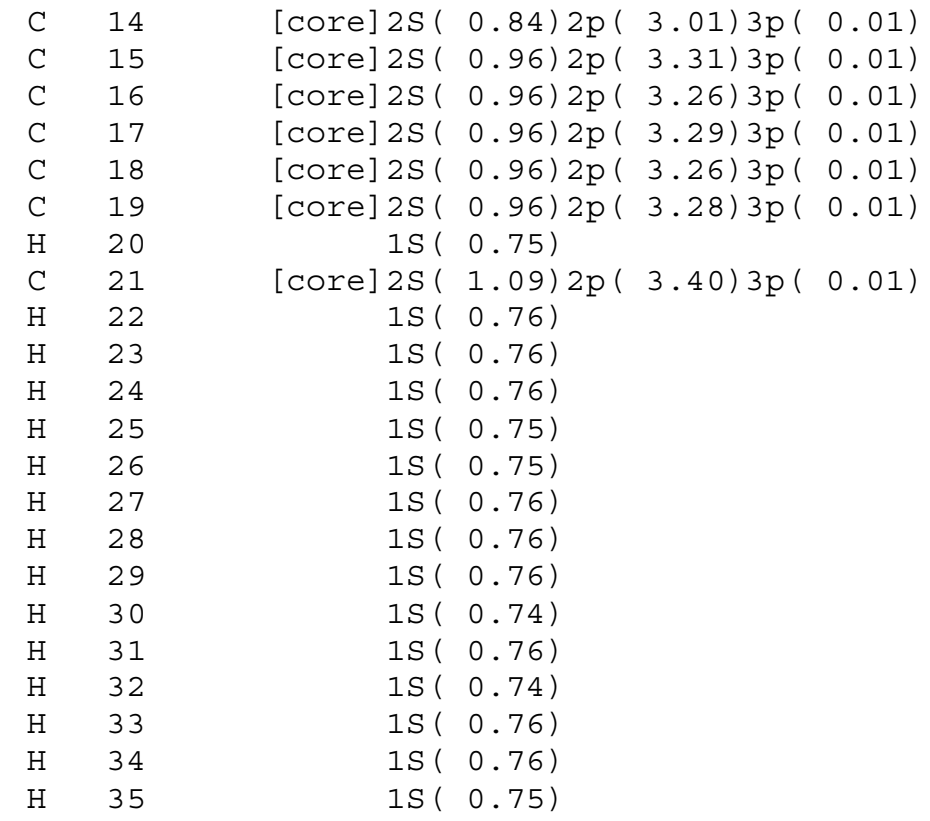

\section{Natural Population Analysis and Natural Electron Configuration for $\left[\mathbf{G a}\left\{\eta^{2}-\mathbf{N}, \mathbf{N}^{\prime}-\right.\right.$ $\left.\left.(\mathbf{P h}) \mathbf{N C}\left(\mathbf{N M e}_{2}\right) \mathbf{N}(\mathbf{P h})\right\}\right]$}

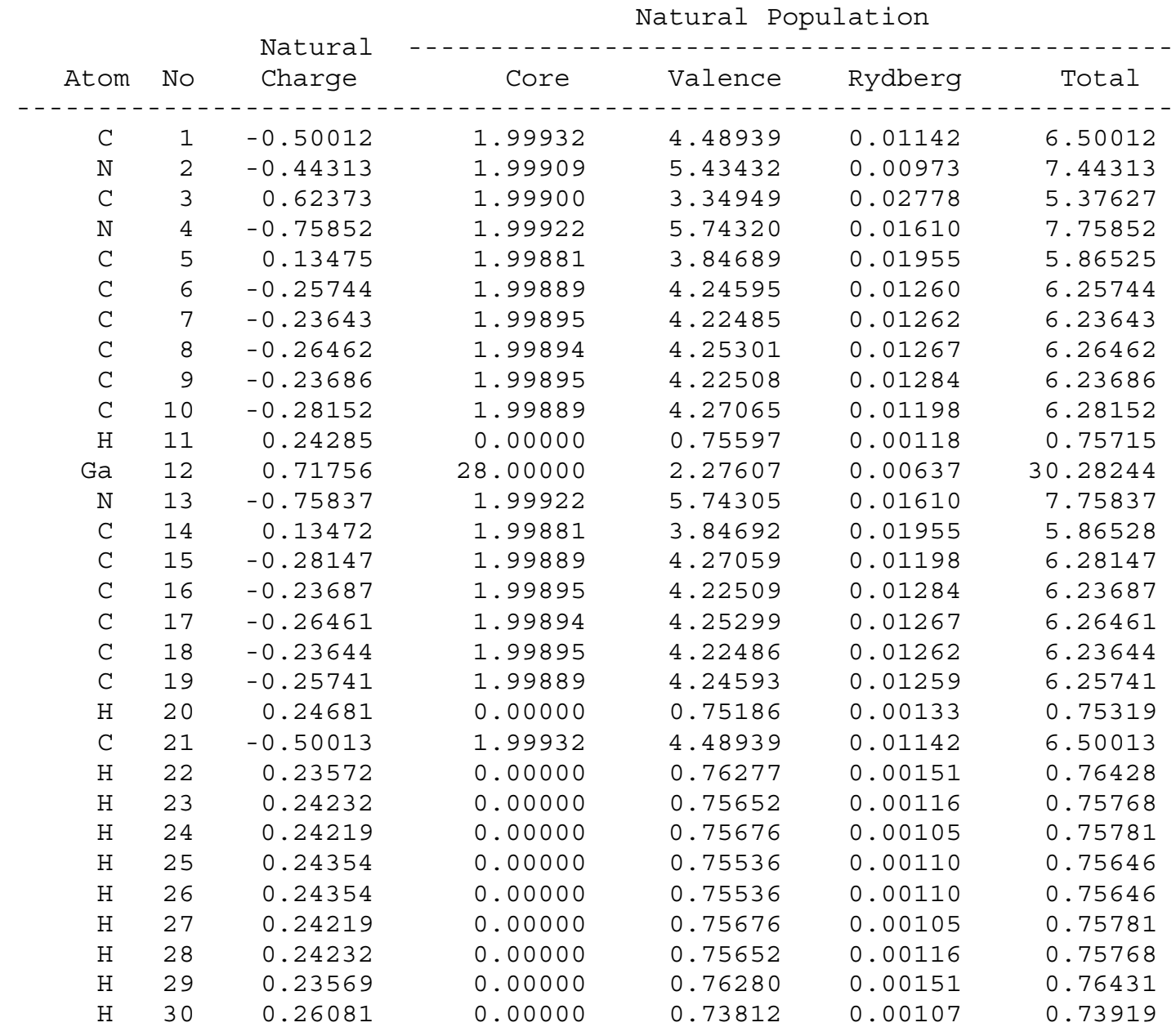




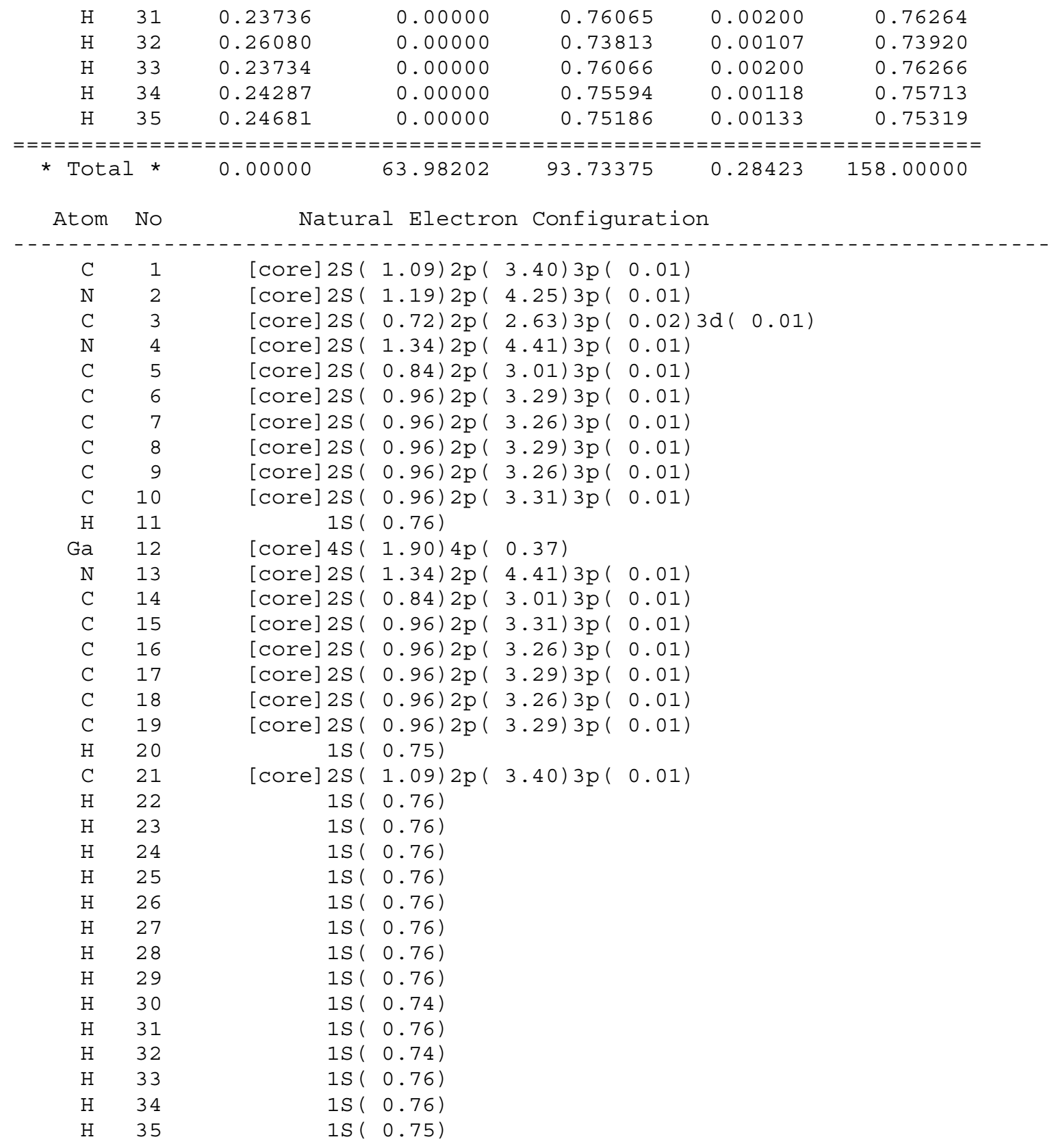

\section{Natural Population Analysis and Natural Electron Configuration for $\left[\operatorname{In}\left\{\eta^{2}-\mathbf{N}, \mathbf{N}^{\prime}-\right.\right.$ $\left.\left.(\mathrm{Ph}) \mathbf{N C}\left(\mathrm{NMe}_{2}\right) \mathbf{N}(\mathbf{P h})\right\}\right]$}

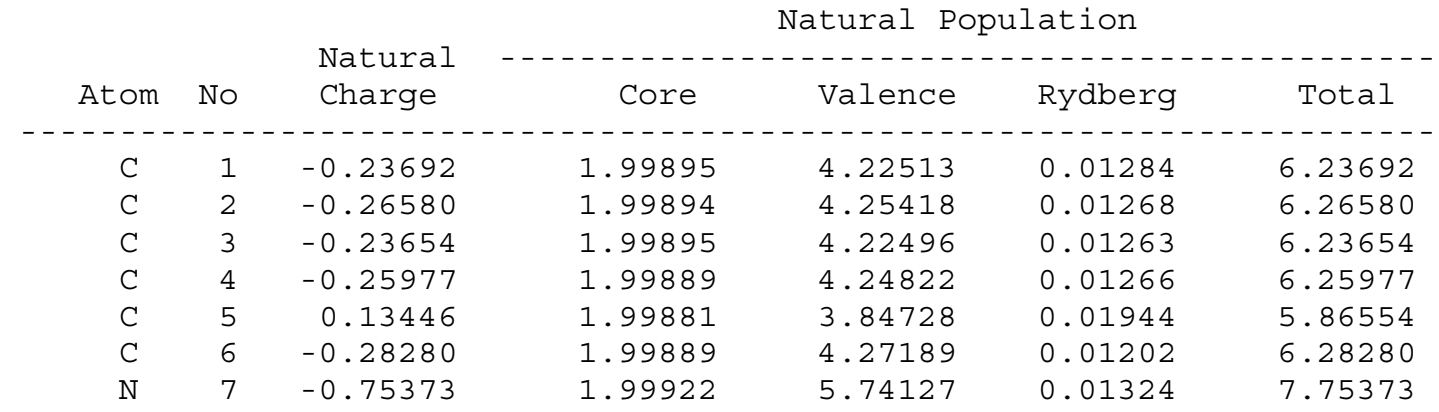




$\begin{array}{rrrrrrr}\mathrm{C} & 8 & 0.61958 & 1.99902 & 3.35394 & 0.02745 & 5.38042 \\ \mathrm{~N} & 9 & -0.44586 & 1.99909 & 5.43709 & 0.00967 & 7.44586 \\ \mathrm{C} & 10 & -0.49993 & 1.99932 & 4.48918 & 0.01144 & 6.49993 \\ \mathrm{In} & 11 & 0.73458 & 46.00000 & 2.26138 & 0.00404 & 48.26542 \\ \mathrm{~N} & 12 & -0.75371 & 1.99922 & 5.74124 & 0.01325 & 7.75371 \\ \mathrm{C} & 13 & 0.13433 & 1.99881 & 3.84741 & 0.01945 & 5.86567 \\ \mathrm{C} & 14 & -0.28273 & 1.99889 & 4.27182 & 0.01202 & 6.28273 \\ \mathrm{C} & 15 & -0.23694 & 1.99895 & 4.22515 & 0.01284 & 6.23694 \\ \mathrm{C} & 16 & -0.26576 & 1.99894 & 4.25415 & 0.01268 & 6.26576 \\ \mathrm{C} & 17 & -0.23654 & 1.99895 & 4.22497 & 0.01262 & 6.23654 \\ \mathrm{C} & 18 & -0.25973 & 1.99889 & 4.24818 & 0.01266 & 6.25973 \\ \mathrm{C} & 19 & -0.49994 & 1.99932 & 4.48919 & 0.01144 & 6.49994 \\ \mathrm{H} & 20 & 0.24209 & 0.00000 & 0.75675 & 0.00116 & 0.75791 \\ \mathrm{H} & 21 & 0.24194 & 0.00000 & 0.75700 & 0.00105 & 0.75806 \\ \mathrm{H} & 22 & 0.24322 & 0.00000 & 0.75567 & 0.00111 & 0.75678 \\ \mathrm{H} & 23 & 0.24322 & 0.00000 & 0.75567 & 0.00111 & 0.75678 \\ \mathrm{H} & 24 & 0.24194 & 0.00000 & 0.75700 & 0.00105 & 0.75806 \\ \mathrm{H} & 25 & 0.24209 & 0.00000 & 0.75675 & 0.00116 & 0.75791 \\ \mathrm{H} & 26 & 0.23466 & 0.00000 & 0.76380 & 0.00154 & 0.76534 \\ \mathrm{H} & 27 & 0.26018 & 0.00000 & 0.73873 & 0.00109 & 0.73982 \\ \mathrm{H} & 28 & 0.23679 & 0.00000 & 0.76119 & 0.00202 & 0.76321 \\ \mathrm{H} & 29 & 0.23471 & 0.00000 & 0.76375 & 0.00154 & 0.76529 \\ \mathrm{H} & 30 & 0.26015 & 0.00000 & 0.73876 & 0.00109 & 0.73985 \\ \mathrm{H} & 31 & 0.23678 & 0.00000 & 0.76120 & 0.00202 & 0.76322 \\ \mathrm{H} & 32 & 0.24286 & 0.00000 & 0.75594 & 0.00120 & 0.75714 \\ \mathrm{H} & 33 & 0.24515 & 0.00000 & 0.75350 & 0.00135 & 0.75485 \\ \mathrm{H} & 34 & 0.24287 & 0.00000 & 0.75594 & 0.00120 & 0.75713 \\ \mathrm{H} & 35 & 0.24510 & 0.00000 & 0.75355 & 0.00135 & 0.75490 \\ \mathrm{TOt} & * & 0.00000 & 81.98205 & 93.74183 & 0.27612 & 176.00000\end{array}$

Atom No Natural Electron Configuration

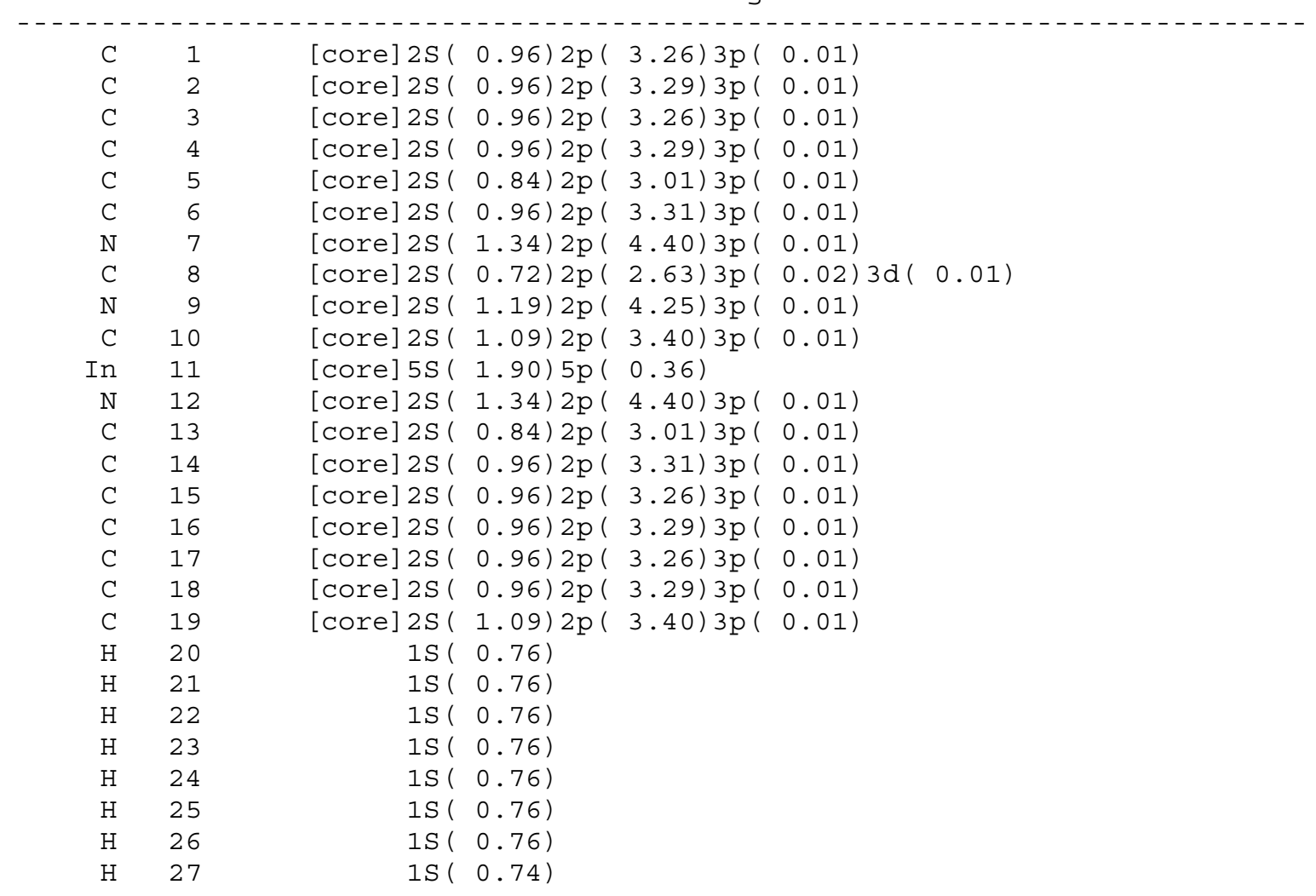




$\begin{array}{lll}\mathrm{H} & 28 & 1 \mathrm{~S}(0.76) \\ \mathrm{H} & 29 & 1 \mathrm{~S}(0.76) \\ \mathrm{H} & 30 & 1 S(0.74) \\ \mathrm{H} & 31 & 1 S(0.76) \\ \mathrm{H} & 32 & 1 S(0.76) \\ \mathrm{H} & 33 & 1 S(0.75) \\ \mathrm{H} & 34 & 1 S(0.76) \\ \mathrm{H} & 35 & 1 S(0.75)\end{array}$

